Research Paper

\title{
Number of Negative Lymph Nodes Can Predict Survival after Postmastectomy Radiotherapy According to Different Breast Cancer Subtypes
}

\author{
San-Gang $\mathrm{Wu}^{1^{*}}$, Fang Peng2 ${ }^{2 *}$, Juan Zhou ${ }^{3^{*}}$, Jia-Yuan Sun ${ }^{4}$, Feng-Yan $\mathrm{Li}^{4}$, Qin Lin ${ }^{1}$, Huan-Xin Lin ${ }^{4}$, Yong \\ $\mathrm{Bao}^{4}$, Zhen-Yu He${ }^{凶}$ \\ 1. Xiamen Cancer Center, Department of Radiation Oncology, the First Affiliated Hospital of Xiamen University, Xiamen 361000, China \\ 2. Department of Radiation Oncology, The First Affiliated Hospital, Sun Yat-sen University, Guangzhou 510060, China \\ 3. Xiamen Cancer Center, Department of Obstetrics and Gynecology, the First Affiliated Hospital of Xiamen University, Xiamen 361000, China \\ 4. Department of Radiation Oncology, Sun Yat-sen University Cancer Center; State Key Laboratory of Oncology in South China; Collaborative \\ Innovation Center of Cancer Medicine, Guangzhou 510080, China \\ * San-Gang Wu, Fang Peng and Juan Zhou contributed equally to this work.
}

$\triangle$ Corresponding author: Yong Bao and Zhen-Yu He. Department of Radiation Oncology, Sun Yat-sen University Cancer Center; State Key Laboratory of Oncology in South China; Collaborative Innovation Center of Cancer Medicine, Guangzhou, China. Tel. +86 20 87343543, Fax. +86 20 87343392, E-mail. hezhy@sysucc.org.cn, and yongbao@sysucc.org.cn

(C) 2015 Ivyspring International Publisher. Reproduction is permitted for personal, noncommercial use, provided that the article is in whole, unmodified, and properly cited. See http://ivyspring.com/terms for terms and conditions.

Received: 2014.08.25; Accepted: 2014.11.15; Published: 2015.01.20

\begin{abstract}
Purpose: To assess the prognostic value of the number of negative lymph nodes (NLNs) in breast cancer patients with positive axillary lymph nodes after mastectomy and its predictive value for radiotherapy efficacy of different breast cancer subtypes (BCS).

Methods: The records of 1,260 breast cancer patients with positive axillary lymph nodes who received mastectomy between January 1998 and December 2007 were reviewed. The prognostic impact and predictive value of the number of NLNs with respect to locoregional recurrence-free survival (LRFS), disease-free survival (DFS), and overall survival (OS) were analyzed.

Results: The median follow-up time was 58 months, and 444 patients (35.2\%) received postmastectomy radiotherapy (PMRT). Univariate and multivariate Cox survival analysis indicated the number of NLNs was an independent prognostic factor of LRFS, DFS, and OS. Patients with a higher number of NLNs had better survival. PMRT improved the LRFS of patients with $\leq 8 \mathrm{NLNs}$ $(p<0.001)$, while failing to improve the LRFS of patients with $>8 \mathrm{NLNs}(p=0.075)$. In patients with luminal A subtype, PMRT improved the LRFS, DFS, and OS of patients with $\leq 8 \mathrm{NLNs}$, but in patients with $>8 \mathrm{NLNs}$ only the LRFS was improved. For patients with luminal B subtype, PMRT only improved the LRFS of patients with $\leq 8 \mathrm{NLNs}$. The number of NLNs had no predictive value for the efficacy with PMRT in Her2+ and triple-negative subtypes.

Conclusions: The number of NLNs is a prognostic indicator in patients with node-positive breast cancer, and it can predict the efficacy of PMRT according to different BCS.
\end{abstract}

Key words: Breast cancer, mastectomy, radiotherapy, negative lymph nodes, prognosis

\section{Introduction}

Meta-analysis has shown that postmastectomy radiotherapy (PMRT) improves the survival of patients of patients with node-positive breast cancer (1).
However, locoregional recurrence (LRR) varies in patients with the same lymph node status, and radiosensitivity may be different due to the heterogeneity 
of breast cancer (2), which affects the efficacy of PMRT. Previous studies have indicated that the breast cancer molecular subtype can predict the efficacy of PMRT (3-6).

While there is a growing number of studies on the replacement of axillary lymph node dissection by sentinel lymph node biopsy (7-10), axillary lymph node status remains an important factor in determining the use of PMRT. The exact assessment of axillary lymph node status is of great relevance to the extent of axillary lymph node dissection, in particular the number of axillary lymph nodes removed $(11,12)$.

In breast cancer patients, the appropriate number of lymph nodes should be removed may be affected by the number of positive lymph nodes. The number of negative lymph nodes (NLNs) is defined as the number of removed lymph nodes minus the number of positive lymph nodes. Differences in number of NLNs may be associated with a different in number of occult lesions. Theoretically, removal of more NLNs reduces the number of occult lesions, thereby improving the survival of patients. If a small number of NLNs are removed, the incidence of LRR may increase due to the presence of occult lesions.

The purpose of PMRT is to reduce the occurrence of LRR, and thus improve survival. The incidence of LRR varies greatly in patients with different breast cancer subtypes (BCS) (13), partly due to different treatment strategies (14) and partly due to the presence of residual lesions (15). Therefore, we hypothesized that patients with different numbers of NLNs have different prognosis, and the number of NLNs may influence the efficacy of PMRT for patients with different BCS. The purpose of this study was to explore the prognostic value of the number of NLNs in patients with node-positive breast cancer after mastectomy, and to evaluate its effects on the efficacy of PMRT in patients with different BCS.

\section{Materials and Methods}

\section{Patients}

The records of patients with breast cancer who were treated at the Sun Yat-sen University Cancer Center (SYSUCC) from January 1998 to December 2007 were retrospectively analyzed. Criteria for inclusion in the analysis were: 1) females with pathologically confirmed unilateral invasive breast cancer; 2) patients who received mastectomy and axillary lymph node dissection, and the number of removed axillary lymph nodes was $\geq 10$; 3) pathological examination confirmed positive axillary lymph nodes and the breast cancer stage was $\mathrm{T} 1-4 \mathrm{~N} 1-3 \mathrm{M} 0$ according to the (2009) 7th edition of the American Joint Committee on Cancer (AJCC)/Union for International Cancer
Control (UICC) staging system; 4) the tumor was completely resected with no positive margins; 5) patients who did not receive neoadjuvant chemotherapy and received at least 4 cycles of postoperative adjuvant chemotherapy; 6) patients who had complete immunohistochemistry results including estrogen receptor (ER), progesterone receptor (PR), and human epidermal growth factor receptor 2 (Her2), and endocrine therapy was administered when indicated. The study was approved by the ethics committee of SYSUCC. All patients provided written consent for storage of their medical information in the hospital database and for research use of this information.

\section{Patient characteristics and lymph node status}

Patients clinicopathological and immunohistochemical factors including age, menstrual status, $\mathrm{pT}$ stage, pN stage, ER, PR, HER2 status, BCS, chemotherapy regimen, and PMRT were used to assess the risks of relapse and death. ER and PR positive was defined as $>1 \%$ positive cells on immunohistochemical staining. Patients were defined as positive for HER2 when immunohistochemistry for HER2 showed $3+$ or $2+$ with confirmation by fluorescence in situ hybridization (FISH). The expression of Ki-67 was determined by immunohistochemical analyses. In SYSUCC before 2008, the expression of Ki-67 was scored by counting the number of positive cells regardless of the staining intensity versus the total number of cells and calculating the percentage of positive cells (positive cells/total cells in one field), as previously described (16), the positivity of several fields were averaged and expressed as the ratio of positive cells per field to total cells per field: $<10 \%$, negative; $10 \%-25 \%$ : weakly positive; $26 \%-50 \%$ : positive; > 50\%: strong positive. A cut-off point of $25 \%$ was used to distinguish between the categories of low and high proliferative tumors. BCS were not determined according to the criteria developed at the St. Gallen International Breast Cancer Conference because some patients did not have Ki-67 immunohistochemistry results (3). And the results of ER, PR, and HER2 were based on the immunohistochemical analysis. Thus, the categorization of BCS was as follows: luminal A (ER+ or PR+, and HER2-), luminal B (ER+ or PR+, and HER2+), HER-2 + (ER-, PR-, and HER2+), and triple negative (TN) (ER-, PR-, and HER2-).

\section{Follow-up and survival endpoints}

Follow-up was performed once every 3 to 6 months. Locoregional recurrence-free survival (LRFS), disease-free survival (DFS), and overall survival (OS) were the primary endpoints. LRR was defined as pathologically confirmed recurrence at the 
ipsilateral chest wall, supraclavicular and subclavian lymph nodes, axillary lymph nodes, or internal mammary lymph nodes. Distant metastasis was defined as recurrence at a site distant from the primary cancer, confirmed by two imaging methods or by pathological assessment. Different examinations were used to confirm potential metastases at distinct sites: bone metastasis required bone scan and magnetic resonance imaging; lung metastasis was usually identified by repeated chest radiograph, followed by chest CT or PET/CT confirmation; for liver metastasis, ultrasound was generally used at follow-up, followed by magnetic resonance imaging or PET/CT if an abnormality was observed. DFS was defined as the absence of locoregional or distant recurrence. OS was calculated as a period of time from the date of diagnosis to the date of death from any cause or the date of last follow-up.

\section{Statistical analysis}

All data were analyzed using the SPSS statistical software package (version 16.0; IBM Corporation, Armonk, NY, USA). The $\chi 2$ and Fisher's exact probability tests were used to analyze the differences between qualitative data. Recognizing that the total number of NLNs may be subject to incomplete counting or natural inter-individual variation in nodal distribution, the number of NLNs was examined as a categorical variable based on quartiles. Survival rates were determined and plotted by the Kaplan-Meier method, and compared using the log rank test. Univariate and multivariate Cox regression model analyses were performed. A value of $p<0.05$ was considered statistically significant.

\section{Results}

\section{Number of NLNs in breast cancer patients}

A total of 1,260 patients were included for analysis, and their characteristics are summarized in Table 1 . The median number of removed lymph nodes was 16 (25th percentile 13, 75th percentile 20; range, 10-73), and the median number of NLNs was 11 (25th percentile 8 , 75th percentile 15 ; range, $0-40$ ). The number of NLNs was examined as a categorical variable based on quartiles: Group $1(0-8, \mathrm{n}=377)$, Group $2(9-11, \mathrm{n}=277)$, Group $3(12-15, \mathrm{n}=325)$, and Group 4 $(16-40, \mathrm{n}=281)$. Table 1 presents the relationships between patient demographics and the number of NLNs. The NLN count was associated with pT stage, $\mathrm{pN}$ stage, ER status, Ki-67 status, and radiation therapy $(p<0.05)$, and was not associated with age, menstrual status, PR status, HER2 status, BCS, and chemotherapy regimen (all, $p>0.05$ ).

\section{Treatment}

A total of 444 patients (35.2\%) underwent PMRT, and the target volume included the ipsilateral chest wall and supra- and infra-clavicular lymph node areas. The radiation dose was 46-50 Gy/23-25 times. The median number of chemotherapy cycles was 6 (range, 4-8), and 1,189 patients (94.4\%) received anthracycline- or taxane-based chemotherapy. A cyclophosphamide (CTX), methotrexate (MTX), and 5-fluorouracil (5-FU) (CMF) regimen was administered in 71 patients $(5.6 \%)$. All patients with positive hormone receptors underwent endocrine therapy; premenopausal patients received tamoxifen (TAM), and postmenopausal patients received TAM or an aromatase inhibitor (AI). No patients who were HER2+ received trastuzumab-targeted therapy.

\section{Survival and disease progression}

The median follow-up time for all patients was 58 months (range, 6-138months). To the date of last follow-up in present study, 979 patients were still alive and the follow-up time was over 5 years in 553 patients (56.5\%). LRR occurred in 151 patients. The details of the LRR events are shown in Table 2. In patients without PMRT, the LRR occurred in 112 patients and the 8-year LRFS rate was $81.5 \%$. PMRT improved LRFS in patients with the 8-year LRFS rates was $89.1 \%(p=0.009)$. The 5 - and 8-year DFS rates were $67.2 \%$ and $60.2 \%$, respectively. A total of 281 patients died among whom 274 died because of breast cancer and 7 died of other diseases. The 5- and 8-year OS rates were $79.2 \%$ and $70.1 \%$, respectively.

\section{Analysis of prognostic factors}

Univariate analysis showed that NLNs as a continuous variable or as a categorical variable was prognostic for LRFS, DFS, and OS (all, $p<0.05$ ). In addition, age, $\mathrm{pT}$ stage, $\mathrm{pN}$ stage, ER status, PR status, HER2 status, BCS and PMRT were factors affecting the survival (all, $p<0.05$ ) (Table 3). The survival curve showing the effect of the number of NLNs on survival is shown in Figure 1.

Multivariate Cox analysis showed that the number of NLNs as a continuous variable was an independent prognostic factor of LRFS (hazard ratio $[\mathrm{HR}]=0.947$, 95\% confidence interval [CI] 0.913-0.981, $p=0.003)$, DFS $(\mathrm{HR}=0.962,95 \%$ CI $0.942-0.982, p<$ 0.022 ), and OS (HR $=0.962,95 \%$ CI $0.937-0.988, p=$ $0.004)$; patients with a higher number of NLNs had better survival. In addition, age, $\mathrm{pT}$ stage, $\mathrm{pN}$ stage, HER2 status, BCS, and PMRT were independent prognostic factors (all, $p<0.05)$ (Table 4 ). 
Table 1. Correlation between number of negative lymph nodes and clinicopathological characteristics.

\begin{tabular}{|c|c|c|c|c|c|c|}
\hline \multirow{3}{*}{ Characteristic } & \multirow{3}{*}{$\mathrm{n}$} & \multicolumn{4}{|c|}{ Number of negative lymph nodes (NLNs) (quartiles) } & \multirow{3}{*}{ P-value } \\
\hline & & 0-8 NLNs & 9-11 NLNs & 12-15 NLNs & 16-40 NLNs & \\
\hline & & $(\mathrm{n}=377)(\%)$ & $(n=277)(\%)$ & $(n=325)(\%)$ & $(\mathrm{n}=281)(\%)$ & \\
\hline \multicolumn{7}{|l|}{ Age (years) } \\
\hline$<35$ & 142 & $35(9.3)$ & $35(12.6)$ & $36(11.1)$ & $36(12.8)$ & 0.443 \\
\hline$\geq 35$ & 1118 & $342(90.7)$ & $242(87.4)$ & $289(88.9)$ & $245(87.2)$ & \\
\hline \multicolumn{7}{|l|}{ Menopausal status } \\
\hline Premenopausal & 838 & $241(63.9)$ & $185(66.8)$ & $219(67.4)$ & $193(68.7)$ & 0.605 \\
\hline Postmenopausal & 422 & $136(36.1)$ & $92(33.2)$ & $106(32.6)$ & $88(31.3)$ & \\
\hline \multicolumn{7}{|l|}{ Histological type } \\
\hline Invasive ductal & 1205 & $362(96.0)$ & $267(96.4)$ & $306(94.1)$ & $270(96.1)$ & 0.744 \\
\hline Invasive lobular & 29 & $6(1.6)$ & $6(2.2)$ & $11(3.4)$ & $6(2.1)$ & \\
\hline Other & 26 & $9(2.4)$ & $4(1.4)$ & $8(2.5)$ & $5(1.8)$ & \\
\hline \multicolumn{7}{|l|}{ Tumor size } \\
\hline $\mathrm{T} 1$ & 309 & $68(18.0)$ & $65(23.5)$ & $91(28.0)$ & $85(30.2)$ & $<0.001$ \\
\hline $\mathrm{T} 2$ & 784 & $218(57.8)$ & $181(65.3)$ & $210(64.6)$ & $175(62.3)$ & \\
\hline $\mathrm{T} 3$ & 111 & $61(16.2)$ & $21(7.6)$ & $15(4.6)$ & $14(5.0)$ & \\
\hline $\mathrm{T} 4$ & 56 & $30(8.0)$ & $10(3.6)$ & $9(2.8)$ & $7(2.5)$ & \\
\hline \multicolumn{7}{|l|}{ Nodal stage } \\
\hline N1 & 655 & $34(9.0)$ & $185(66.8)$ & $224(68.9)$ & $212(75.4)$ & $<0.001$ \\
\hline N2 & 321 & $129(34.2)$ & $63(22.7)$ & $75(23.1)$ & $54(19.2)$ & \\
\hline N3 & 284 & $214(56.8)$ & $29(10.5)$ & $26(8)$ & $15(5.4)$ & \\
\hline \multicolumn{7}{|l|}{ ER status } \\
\hline Negative & 536 & $185(49.1)$ & $108(39.0)$ & $131(40.3)$ & $112(39.9)$ & 0.023 \\
\hline Positive & 724 & $192(50.9)$ & $169(61.0)$ & $194(59.7)$ & $169(60.1)$ & \\
\hline \multicolumn{7}{|l|}{ PR status } \\
\hline Negative & 445 & 149 (39.5) & $88(31.8)$ & 103 (31.7) & $105(37.4)$ & 0.077 \\
\hline Positive & 815 & $228(60.5)$ & $189(68.2)$ & $222(68.3)$ & $176(62.6)$ & \\
\hline \multicolumn{7}{|l|}{ HER2 status } \\
\hline Negative & 800 & $243(64.5)$ & $167(60.3)$ & $215(66.2)$ & $175(62.3)$ & 0.466 \\
\hline Positive & 460 & $134(35.5)$ & $110(39.7)$ & $110(33.8)$ & $106(37.7)$ & \\
\hline \multicolumn{7}{|l|}{ Ki-67 } \\
\hline$\leq 25 \%$ positive & 514 & $125(33.2)$ & $119(43.0)$ & $141(43.4)$ & $129(45.9)$ & $<0.001$ \\
\hline$>25 \%$ positive & 273 & $83(22.0)$ & 49 (17.7) & $60(18.5)$ & $81(28.8)$ & \\
\hline Unknown & 473 & $169(44.8)$ & $109(39.3)$ & $124(38.1)$ & $71(25.3)$ & \\
\hline \multicolumn{7}{|l|}{ Breast cancer subtypes } \\
\hline Luminal A & 635 & $183(48.6)$ & $135(48.7)$ & $175(53.8)$ & $142(50.6)$ & 0.148 \\
\hline Luminal B & 262 & $74(19.6)$ & $74(26.7)$ & $60(18.5)$ & $54(19.2)$ & \\
\hline HER2+ & 198 & $60(15.9)$ & $36(13.0)$ & $50(15.4)$ & $52(18.5)$ & \\
\hline Triple-negative & 165 & $60(15.9)$ & $32(11.6)$ & $40(12.3)$ & $33(11.7)$ & \\
\hline \multicolumn{7}{|l|}{ Chemotherapy } \\
\hline $\mathrm{CMF}$ & 71 & $24(6.4)$ & $17(6.1)$ & $17(5.2)$ & $13(4.6)$ & 0.766 \\
\hline Taxane and/or anthracycline & 1189 & $353(93.6)$ & $260(93.9)$ & $308(94.8)$ & $268(95.4)$ & \\
\hline \multicolumn{7}{|l|}{ PMRT } \\
\hline No & 816 & $142(37.7)$ & $204(73.6)$ & $247(76)$ & $223(79.4)$ & $<0.001$ \\
\hline Yes & 444 & $235(62.3)$ & $73(26.4)$ & $78(24)$ & $58(20.6)$ & \\
\hline
\end{tabular}

ER, estrogen receptor; PR, progesterone receptor; HR, hormone receptor; CMF, cyclophosphamide, methotrexate, and 5-fluorouracil; PMRT, postmastectomy radiotherapy.

Table 2. Distribution of the events by specific locoregional recurrence site.

\begin{tabular}{llll}
\hline Locoregional recurrence site & $\mathrm{n}(\mathrm{n}=151)(\%)$ & Without PMRT $(\mathrm{n}=112)(\%)$ & With PMRT(n=39) $(\%)$ \\
\hline Isolated chest wall & $48(31.8)$ & $32(28.6)$ & $16(41.0)$ \\
Isolated supraclavicular lymph nodes & $62(41.1)$ & $55(49.1)$ & $7(18.0)$ \\
Isolated infraclavicular lymph nodes & $2(1.3)$ & $2(1.8)$ & $0(0)$ \\
Isolated axillary lymph nodes & $6(4.0)$ & $3(2.7)$ & $3(7.7)$ \\
Isolated internal mammary lymph nodes & $2(1.3)$ & $2(1.7)$ & $0(0)$ \\
Multiple sites & $31(20.5)$ & $18(16.1)$ & $13(33.3)$ \\
\hline
\end{tabular}

PMRT, postmastectomy radiotherapy. 
Table 3. Univariate analysis of prognostic factors.

\begin{tabular}{|c|c|c|c|c|c|c|c|c|c|}
\hline & LRFS & & & DFS & & & OS & & \\
\hline Characteristic & HR & $95 \% \mathrm{CI}$ & P-value & HR & $95 \% \mathrm{CI}$ & P-value & HR & $95 \% \mathrm{CI}$ & $\mathrm{P}$-value \\
\hline \multicolumn{10}{|l|}{ Age (years) } \\
\hline$<35$ & 1 & & & 1 & & & 1 & & \\
\hline$\geq 35$ & 0.765 & $0.482-1.213$ & 0.254 & 0.717 & $0.543-0.947$ & 0.019 & 0.780 & $0.556-1.097$ & 0.150 \\
\hline \multicolumn{10}{|l|}{ Menopausal status } \\
\hline Premenopausal & 1 & & & 1 & & & 1 & & \\
\hline Postmenopausal & 0.854 & $0.603-1.211$ & 0.377 & 0.975 & $0.792-1.200$ & 0.811 & 1.049 & $0.818-1.344$ & 0.707 \\
\hline \multicolumn{10}{|l|}{ Histological type } \\
\hline Invasive ductal & 1 & & & 1 & & & 1 & & \\
\hline Invasive lobular & 0.524 & $0.130-2.115$ & 0.364 & 0.560 & $0.250-1.254$ & 0.159 & 0.561 & $0.209-1.505$ & 0.251 \\
\hline Other & 0.946 & $0.301-2.967$ & 0.924 & 0.926 & $0.460-1.865$ & 0.829 & 0.493 & $0.158-1.537$ & 0.223 \\
\hline \multicolumn{10}{|l|}{ Tumor size } \\
\hline $\mathrm{T} 1$ & 1 & & & & 1 & & 1 & & \\
\hline $\mathrm{T} 2$ & 1.338 & $0.876-2.043$ & 0.177 & 1.412 & $1.090-1.830$ & 0.009 & 1.505 & $1.090-2.078$ & 0.013 \\
\hline $\mathrm{T} 3$ & 2.303 & $1.307-4.055$ & 0.004 & 2.481 & $1.753-3.512$ & $<0.001$ & 2.689 & $1.768-4.088$ & $<0.001$ \\
\hline $\mathrm{T} 4$ & 2.451 & $1.191-5.047$ & 0.015 & 2.521 & $1.612-3.942$ & $<0.001$ & 2.830 & $1.676-4.777$ & $<0.001$ \\
\hline \multicolumn{10}{|l|}{ Nodal stage } \\
\hline N1 & 1 & & & 1 & & & 1 & & \\
\hline N2 & 1.321 & $0.880-1.983$ & 0.180 & 1.765 & $1.376-2.263$ & $<0.001$ & 1.930 & $1.416-2.630$ & $<0.001$ \\
\hline N3 & 2.518 & $1.739-3.646$ & $<0.001$ & 3.588 & $2.853-4.513$ & $<0.001$ & 4.200 & $3.179-5.551$ & $<0.001$ \\
\hline \multicolumn{10}{|l|}{ ER status } \\
\hline Negative & 1 & & & 1 & & & 1 & & \\
\hline Positive & 0.517 & $0.375-0.713$ & $<0.001$ & 0.549 & $0.452-0.667$ & $<0.001$ & 0.510 & $0.403-0.646$ & $<0.001$ \\
\hline \multicolumn{10}{|l|}{ PR status } \\
\hline Negative & 1 & & & 1 & & & 1 & & \\
\hline Positive & 0.576 & $0.418-0.793$ & 0.001 & 0.603 & $0.496-0.733$ & $<0.001$ & 0.526 & $0.417-0.665$ & $<0.001$ \\
\hline \multicolumn{10}{|l|}{ HER2 status } \\
\hline Negative & 1 & & & 1 & & & 1 & & \\
\hline Positive & 1.539 & $1.117-2.120$ & 0.008 & 1.472 & $1.210-1.791$ & $<0.001$ & 1.334 & $1.053-1.690$ & 0.017 \\
\hline \multicolumn{10}{|l|}{$\mathrm{Ki}-67^{*}$} \\
\hline$\leq 25 \%$ positive & 1 & & & 1 & & & 1 & & \\
\hline$>25 \%$ positive & 0.865 & $0.536-1.398$ & 0.554 & 1.243 & $0.934-1.654$ & 0.135 & 1.345 & $0.948-1.908$ & 0.096 \\
\hline \multicolumn{10}{|l|}{ Breast cancer subtypes } \\
\hline Luminal A & 1 & & & 1 & & & 1 & & \\
\hline Luminal B & 1.583 & $1.029-2.435$ & 0.037 & 1.476 & $1.142-1.907$ & 0.003 & 1.295 & $0.939-1.786$ & 0.115 \\
\hline HER2+ & 2.455 & $1.602-3.763$ & $<0.001$ & 2.087 & $1.604-2.715$ & $<0.001$ & 2.205 & $1.612-3.015$ & $<0.001$ \\
\hline Triple-negative & 2.341 & $1.488-3.682$ & $<0.001$ & 1.906 & $1.438-2.528$ & $<0.001$ & 2.207 & $1.594-3.055$ & $<0.001$ \\
\hline \multicolumn{10}{|l|}{ Chemotherapy } \\
\hline $\mathrm{CMF}$ & 1 & & & 1 & & & 1 & & \\
\hline Taxane and/or anthracycline & 0.840 & $0.455-1.554$ & 0.580 & 0.762 & $0.531-1.088$ & 0.135 & 0.912 & $0.589-1.414$ & 0.681 \\
\hline \multicolumn{10}{|l|}{ PMRT } \\
\hline No & 1 & & & 1 & & & 1 & & \\
\hline Yes & 0.619 & $0.430-0.891$ & 0.010 & 1.176 & $0.933-1.436$ & 0.111 & 1.268 & 0.999-1.609 & 0.051 \\
\hline Number of NLNs (continuous) & 0.930 & $0.903-0.958$ & $<0.001$ & 0.919 & $0.902-0.936$ & $<0.001$ & 0.906 & $0.886-0.927$ & $<0.001$ \\
\hline \multicolumn{10}{|l|}{ Number of NLNs (categorical) } \\
\hline $0-8$ & 1 & & & 1 & & & 1 & & \\
\hline $9-11$ & 0.638 & 0.424-0.959 & 0.031 & 0.489 & $0.376-0.634$ & $<0.001$ & 0.312 & $0.221-0.442$ & $<0.001$ \\
\hline $12-15$ & 0.531 & $0.351-0.805$ & 0.003 & 0.498 & $0.388-0.639$ & $<0.001$ & 0.458 & $0.341-0.616$ & $<0.001$ \\
\hline $16-40$ & 0.296 & $0.171-0.512$ & $<0.001$ & 0.308 & $0.225-0.421$ & $<0.001$ & 0.297 & $0.203-0.433$ & $<0.001$ \\
\hline
\end{tabular}

LRFS, locoregional recurrence-free survival; DFS, disease-free survival; OS, overall survival; CI, confidence interval; ER, estrogen receptor; PR, progesterone receptor; HR, hormone receptor; CMF, cyclophosphamide, methotrexate, and 5-fluorouracil; PMRT, postmastectomy radiotherapy; NLNs, negative lymph nodes.

* With missing data.

Table 4. Multivariate analysis of prognostic factors.

\begin{tabular}{|c|c|c|c|c|c|c|c|c|c|}
\hline & LRFS & & & DFS & & & OS & & \\
\hline Characteristics & HR & $95 \% \mathrm{CI}$ & P-value & HR & $95 \% \mathrm{CI}$ & P-value & HR & $95 \% \mathrm{CI}$ & P-value \\
\hline Age & - & & & 0.708 & $0.536-0.935$ & 0.015 & - & & \\
\hline Tumor size & 1.325 & $1.074-1.634$ & 0.009 & 1.203 & $1.055-1.371$ & 0.006 & 1.255 & $1.078-1.460$ & 0.003 \\
\hline Nodal stage & 0.644 & $1.287-2.100$ & $<0.001$ & 1.585 & $1.373-1.831$ & $<0.001$ & 1.680 & $1.407-2.006$ & $<0.001$ \\
\hline ER status & 0.839 & $0.532-1.322$ & 0.448 & 0.822 & $0.631-1.072$ & 0.149 & 0.856 & $0.616-1.189$ & 0.354 \\
\hline PR status & 1.233 & $0.712-2.135$ & 0.454 & 0.983 & $0.717-1.348$ & 0.915 & 0.834 & $0.579-1.202$ & 0.331 \\
\hline HER2 status & 1.267 & $0.912-1.760$ & 0.159 & 1.330 & $1.086-1.629$ & 0.006 & 1.211 & $0.950-1.545$ & 0.122 \\
\hline Breast cancer subtypes & 1.354 & $1.183-1.549$ & $<0.001$ & 1.238 & $1.132-1.354$ & $<0.001$ & 1.331 & $1.206-1.469$ & $<0.001$ \\
\hline PMRT & 0.297 & $0.198-0.444$ & $<0.001$ & - & & & - & & \\
\hline Number of NLNs (continuous) & 0.947 & $0.913-0.981$ & 0.003 & 0.962 & $0.942-0.982$ & $<0.001$ & 0.962 & $0.937-0.988$ & 0.004 \\
\hline
\end{tabular}

LRFS, locoregional recurrence-free survival; DFS, disease-free survival; OS, overall survival; CI, confidence interval; ER, estrogen receptor; PR, progesterone receptor; HR, hormone receptor; PMRT, postmastectomy radiotherapy; NLNs, negative lymph nodes. 


\section{Effect of the number of NLNs on the LRFS of patients who did not receive PMRT}

The subgroup analysis of 816 patients who did not receive PMRT showed that the effect of the number of NLNs on LRFS was significantly different among groups. The 8-year LRFS in Group 1, Group 2, Group 3, and Group 4 were $62.5 \%, 81.7 \%, 84.3 \%$, and $90.5 \%$, respectively $(p<0.001)$. The survival curves of Group 2, Group 3, and Group 4 crossed and overlapped. Therefore, the 3 groups were combined, and the analysis showed that the survival of patients with $>8$ NLNs was significant better than that of patients with $\leq 8$ NLNs. The 8 -year LRFS were 85.2 and $62.5 \%$ in patients with $>8$ NLNs and $\leq 8$ NLNs, respectively $(p<0.001)$.

\section{Effect of the number of NLNs in LRR with PMRT}

PMRT improved the LRFS of patients with $\leq 8$ NLNs, 8-year LRFS rates of patients who with and without PMRT were $84.4 \%$ and $62.5 \%$, respectively ( $p$ $<0.001$ ) (Figure 2A). For patients with > 8 NLNs, PMRT did not improve the LRFS. The 8-year LRFS rates in patients with and without PMRT 93.2\% and $85.2 \%$, respectively $(p=0.075)$ (Figure $2 \mathrm{~B}$ ).

\section{Effect of the number of NLNs on the efficacy of PMRT according to different BCS}

Subgroup analysis showed that PMRT improved the LRFS, DFS, and OS of luminal A subtype with $\leq 8$ NLNs (Figure 3A-3C). For patients with $>8$ NLNs, PMRT improved the LRFS but did not affect DFS and OS. PMRT improved the LRFS of luminal B subtype with $\leq 8$ NLNs, but did not affect the DFS and OS. The number of NLNs did not affect the efficacy of PMRT in Her2+ and triple-negative subtypes.

Table 5. Effect of the number of negative lymph nodes on survival after radiotherapy in patients with different breast cancer subtypes.

\begin{tabular}{|c|c|c|c|c|c|c|}
\hline Breast cancer subtypes & 0-8 NLNs (8 year) & & & 9-40 NLNs (8 year) & & \\
\hline & $\begin{array}{l}\text { Without } \\
\text { PMRT }\end{array}$ & With PMRT & P-value & Without PMRT & With PMRT & P-value \\
\hline \multicolumn{7}{|l|}{ Luminal A } \\
\hline LRFS & 63.2 & 91.6 & $<0.001$ & 87.2 & 98.0 & 0.019 \\
\hline DFS & 40.2 & 54.7 & 0.002 & 73.6 & 53.9 & 0.407 \\
\hline OS & 50.8 & 75.8 & 0.008 & 83.1 & 75.7 & 0.227 \\
\hline \multicolumn{7}{|l|}{ Luminal B } \\
\hline LRFS & 51.8 & 75.4 & 0.007 & 88 & 93.6 & 0.404 \\
\hline DFS & 30.6 & 44.7 & 0.196 & 67.1 & 70.7 & 0.667 \\
\hline OS & 50.4 & 49.3 & 0.252 & 82.2 & 82.6 & 0.850 \\
\hline \multicolumn{7}{|l|}{ HER2+ } \\
\hline LRFS & 71.1 & 84.2 & 0.245 & 78.4 & 85.2 & 0.794 \\
\hline DFS & 36.6 & 24.8 & 0.659 & 58.3 & 76.8 & 0.270 \\
\hline Os & 19.4 & 35.5 & 0.852 & 67.4 & 84.8 & 0.119 \\
\hline \multicolumn{7}{|l|}{ Triple-negative } \\
\hline LRFS & 62.7 & 75.3 & 0.293 & 81.2 & 81.7 & 0.738 \\
\hline DFS & 41.2 & 31.6 & 0.989 & 60.8 & 58.7 & 0.671 \\
\hline Os & 47.1 & 32.4 & 0.934 & 69.7 & 53.1 & 0.386 \\
\hline
\end{tabular}

LRFS, locoregional recurrence-free survival; DFS, disease-free survival; OS, overall survival; PMRT, postmastectomy radiotherapy; NLNs, negative lymph nodes.
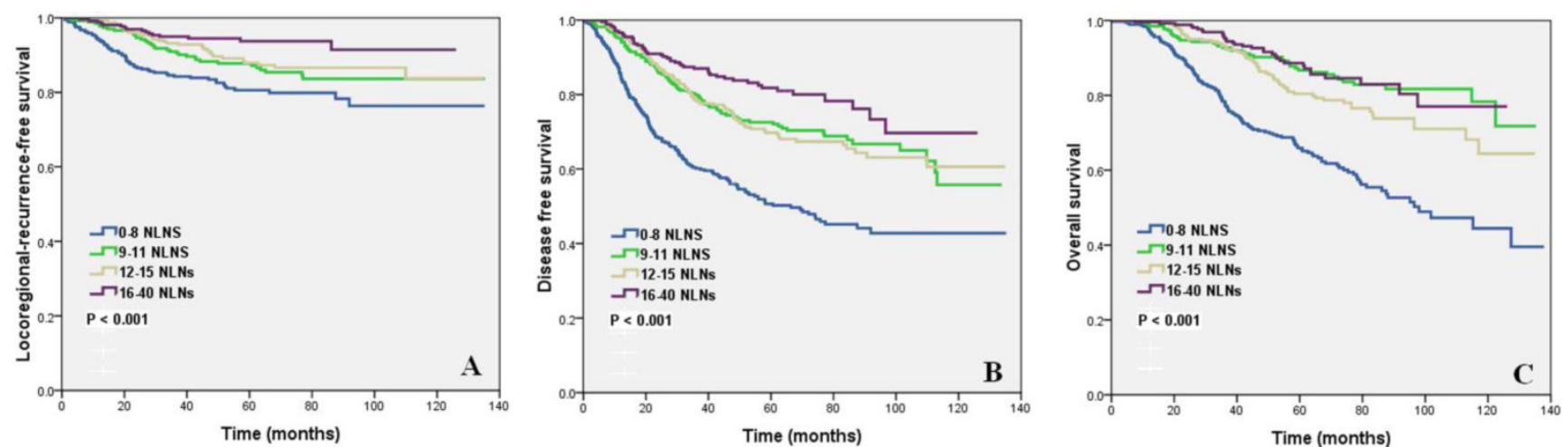

Figure 1. Impact of the number of negative lymph nodes on locoregional recurrence-free survival (A), disease-free survival (B) and overall survival (C). 

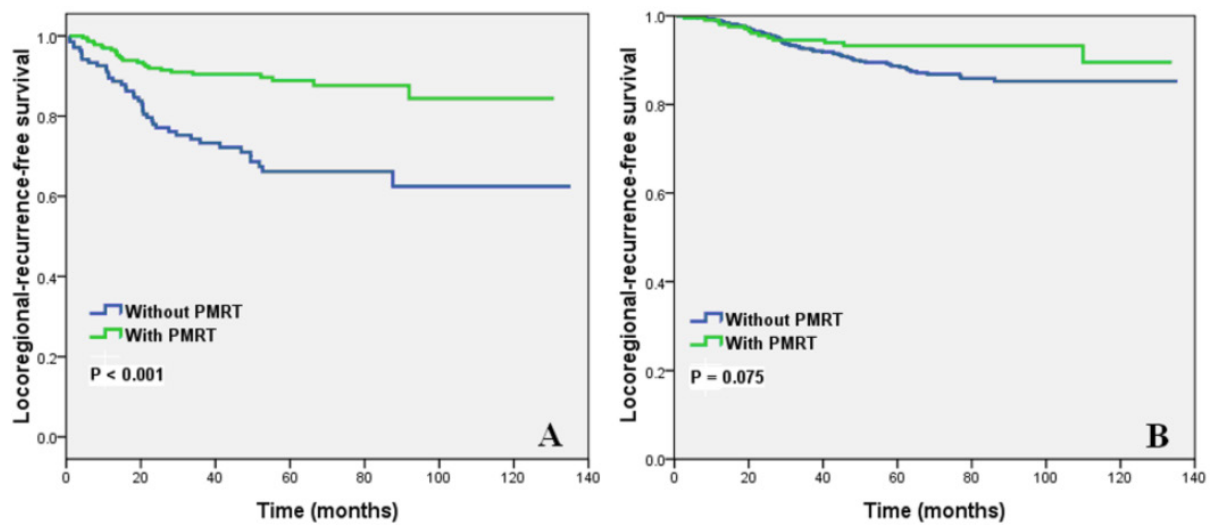

Figure 2. Impact of the number of negative lymph nodes on locoregional recurrence-free survival (A, 0-8 NLNs; B, 9-40 NLNs) of patients with and without PMRT.
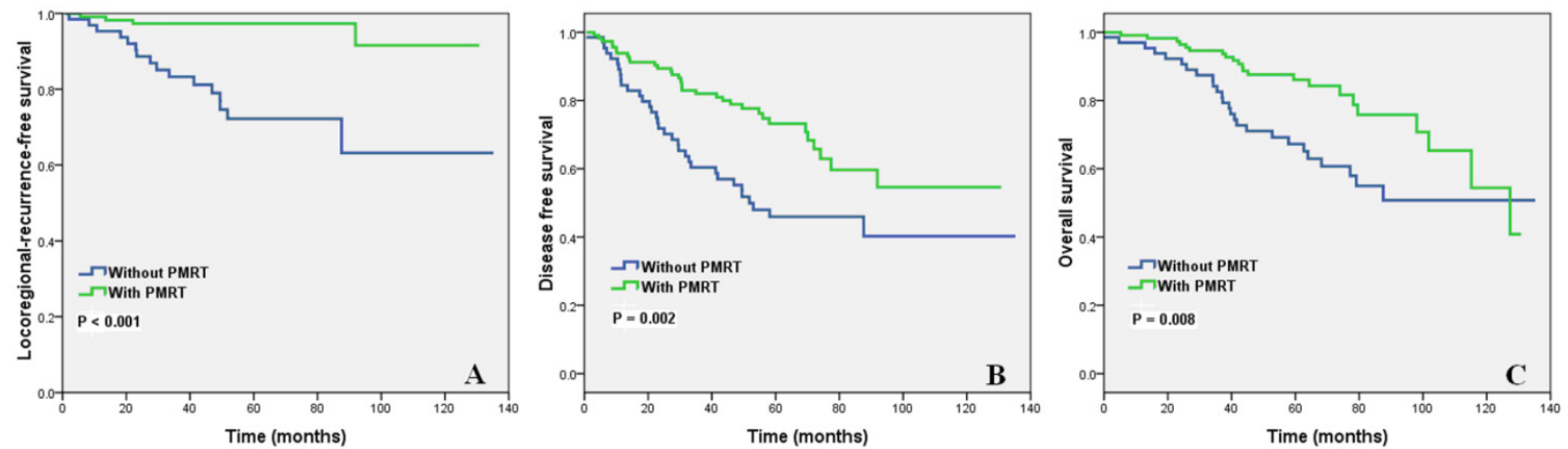

Figure 3. Impact of PMRT on locoregional recurrence-free survival (A), disease-free survival (B) and overall survival (C) of patients with 0-8 NLNs in luminal A subtype.

\section{Discussion}

The present study assessed the prognostic value of NLN count in patients with node-positive breast cancer after mastectomy, and the effects of the number of NLNs on the efficacy of PMRT for different BCS. The results showed that the number of NLNs is an independent prognostic factor in breast cancer survival, and NLN count can be used to predict the efficacy of PMRT in patients with different BCS.

Survival after sentinel lymph node biopsy and axillary lymph node dissection is similar in specific populations with breast cancer $(7,8)$, and sentinel lymph node biopsy has an advantage in that it is associated with reduced postoperative lymphedema $(9$, 10). However, axillary lymph node status is still one of the most important prognostic indicators in breast cancer, especially in patients with positive axillary lymph nodes. As the dissection of more NLN count may reduce the number of occult lesions and improve the prognosis, the number of NLNs may better reflect the extent of axillary lymph node dissection.

The prognostic value of NLN count has been confirmed in esophageal, rectal, and cervical cancer
(17-19). However, studies on the prognostic value of NLN count are still limited (20, 21). Karlsson et al. (20) showed that the number of NLNs was an independent prognostic factor of LRFS and OS of patients who did not undergo PMRT. Patients with $\geq 10$ NLNs had a significantly better prognosis than patients with $<10$ NLNs, especially in patients with positive lymph nodes. Nevertheless, it would not affect the prognosis of patients with node-negative disease (20). In a study in which $68 \%$ of patients received PMRT, patients with >15 NLNs had better OS (12). However, the above studies were limited because adjuvant chemotherapy and endocrine therapy were either insufficient or not clearly stated. In present study, we found that the number of NLNs was an important prognostic factor, but PMRT did not benefit patients with higher number of NLNs. This may be because patients with fewer NLNs have more occult lesions, and the primary objective of PMRT is to eliminate locoregional residue lesions and improve locoregional control. Therefore, radiotherapy may be benefits in patients with fewer NLNs.

Individualized treatment is the goal of comprehensive treatment of breast cancer. Gentilini et al. (2) 
performed level I to III complete axillary lymph node dissection, and the median number of removed lymph nodes was 23 . The 5-year LRR rates of patients who were hormone receptor positive and with node-negative, $1-3$ positive nodes, and $\geq 4$ positive lymph nodes were $2.3 \%, 7.6 \%$, and $7.6 \%$, respectively, while the LRR rates of patients in the same lymph node categories but who were hormone receptor negative were $5.9 \%, 10.3 \%$, and $20 \%$, respectively. It suggests that hormone receptor status and the number of positive lymph nodes can be used to determine prognosis and thus influence the selection of adjuvant treatment.

There are different therapeutic strategies for different BCS (3). In addition, studies have shown that different BCS have different radiosensitivities (4-6). For this reason, we further conducted BCS analysis and the results showed that the number of NLNs could predict the efficacy of PMRT for different BCS, especially in luminal A subtype patients. This result suggests that for luminal A subtype patients with a better prognosis, an adequate number of NLNs can further reduce the number of occult lesions and achieve a better locoregional control, thereby making it possible to avoid radiation therapy. It is believed that HER2+ and TN breast cancers may be radiation resistant $(21,22)$. In this study, PMRT did not benefit patients with HER2+ and TN breast cancer regardless of the number of NLNs. However, the survival of patients with a higher number of NLNs was superior to that of patients with a fewer number of NLNs. This also suggests that when the number of NLNs is higher, the number of occult lesions is reduced, thereby improving survival.

We need to recognize the limitations of the present study. First, this was a single center retrospective study, and thus cannot represent the population at large. Patients with HER2+ breast cancer did not routine undergo trastuzumab treatment, which may affect the results. In addition, the optimal cut-off point of number of NLNs is not consistent with the previously findings $(12,20)$. This might be ascribed to differences in the clinicopathological characteristics, surgical modalities, and methods used for statistical analysis. In future prospective multicenter studies, it will be necessary to confirm the specific value of the number of NLNs in breast cancer patients and to explore the optimal cut-off point. Third, the number of NLNs is different according to the pathologist and to surgeon. Frequently, surgeons resect fragments of a lymph node for which pathologists assign duplicate or multiple counts for the same node.

\section{Conclusion}

Although the value of the number of NLNs in patients with breast cancer requires further study, the current results suggest that the number of NLNs is an important prognostic indicator for patients with node-positive breast cancer, and it can predict the efficacy of PMRT of different BCS. Patients with a higher number of NLNs have better survival and may not require PMRT. More studies are required to confirm our findings and to investigate the related mechanisms.

\section{Abbreviations}

PMRT: postmastectomy radiotherapy; LRR: locoregional recurrence; BCS: breast cancer subtypes; NLNs: negative lymph nodes; AJCC: American Joint Committee on Cancer; UICC: Union for International Cancer Control; ER: estrogen receptor; PR: progesterone receptor; Her2: human epidermal growth factor receptor 2; TN: triple negative; FISH: fluorescent in situ hybridization; LRFS: locoregional recurrence-free survival; DFS: disease-free survival; OS: overall survival; CMF: cyclophosphamide, methotrexate, and 5-fluorouracil; TAM: tamoxifen; HR: hazard ratio; CI: confidence interval.

\section{Acknowledgments}

This study was supported by a grant from the Sci-Tech Office of Guangdong Province (No. 2013B021800157), Medical Scientific Research Foundation of Guangdong Province (A2010192), the Youth Foundation of the First Affiliated Hospital of Xiamen University (No. XYY2012005) and the Education Scientific Research Project of Young Teachers in Fujian Province (No. JB13131).

\section{Competing Interests}

The authors have declared that no competing interest exists.

\section{References}

1. EBCTCG (Early Breast Cancer Trialists' Collaborative Group), McGale P, Taylor C, et al. Effect of radiotherapy after mastectomy and axillary surgery on 10-year recurrence and 20-year breast cancermortality: meta-analysis of individual patient data for 8135 women in 22 randomised trials. Lancet. 2014;383(9935):2127-35.

2. Gentilini O, Botteri $\mathrm{E}$, Rotmensz N, et al. Is avoiding post-mastectomy radiotherapy justified for patients with four or more involved axillary nodes andendocrine-responsive tumours? Lessons from a series in a single institution. Ann Oncol. 2007;18(8):1342-7.

3. Goldhirsch A, Winer EP, Coates AS, et al. Personalizing the treatment of women with early breast cancer: highlights of the St Gallen International Expert Consensus on the Primary Therapy of Early Breast Cancer 2013. Ann Oncol. 2013;24(9):2206-23.

4. Wu SG, He ZY, Li Q, et al. Predictive value of breast cancer molecular subtypes in Chinese patients with four or more positive nodes after postmastectomy radiotherapy. Breast. 2012;21(5):657-61.

5. Kyndi M, Sorensen FB, Knudsen H, Overgaard M, Nielsen HM, Overgaard J. Estrogen receptor, progesterone receptor, HER-2, and response to post-mastectomy radiotherapy in high-risk breast cancer: the Danish breast cancer cooperative group. J Clin Oncol. 2008;26(9):1419-26.

6. Wang SL, Li YX, Song YW, et al. Triple-negative or HER2-positive status predicts higher rates of locoregional recurrence in node-positive breast cancer patients after mastectomy. Int J Radiat Oncol Biol Phys. 2011;80(4):1095-101. 
7. Giuliano AE, McCall L, Beitsch P, et al. Locoregional recurrence after sentinel lymph node dissection with or without axillary dissection in patients with sentinel lymph node metastases: the American College of Surgeons Oncology Group Z0011 randomized trial. Ann Surg. 2010;252(3):426-32; discussion 432-3.

8. Giuliano AE, Hunt KK, Ballman KV, et al. Axillary dissection vs no axillary dissection in women with invasive breast cancer and sentinel node metastasis: a randomized clinical trial. JAMA. 2011;305(6):569-75.

9. Sackey H, Magnuson A, Sandelin K, et al. Arm lymphoedema after axillary surgery in women with invasive breast cancer. Br J Surg. 2014;101(4):390-7.

10. De Gournay E, Guyomard A, Coutant C, et al. Impact of sentinel node biopsy on long-term quality of life in breast cancer patients. $\mathrm{Br} \mathrm{J}$ Cancer. 2013;109(11):2783-91.

11. Somner JE, Dixon JM, Thomas JS. Node retrieval in axillary lymph node dissections: recommendations for minimum numbers to be confident about node negative status. J Clin Pathol. 2004;57(8):845-8.

12. Kuru B. Prognostic significance of total number of nodes removed, negative nodes removed, and ratio of positive nodes to removed nodes in node positive breast carcinoma. Eur J Surg Oncol. 2006;32(10):1082-8.

13. Sioshansi S, Huber KE, Wazer DE. The implications of breast cancer molecular phenotype for radiation oncology. Front Oncol. 2011;1:12.

14. Curigliano G. New drugs for breast cancer subtypes: targeting driver pathways to overcome resistance. Cancer Treat Rev. 2012;38(4):303-10.

15. Sioshansi S, Ehdaivand S, Cramer C, Lomme MM, Price LL, Wazer DE. Triple negative breast cancer is associated with an increased risk of residual invasive carcinoma after lumpectomy. Cancer. 2012;118(16):3893-8.

16. Li BJ, Zhu ZH, Wang JY, et al. [Expression correlation of Ki67 to P53, VEGF, and C-erbB-2 genes in breast cancer and their clinical significances]. Ai Zheng. 2004;23(10):1176-9. [Article in Chinese]

17. Hsu PK, Huang CS, Wang BY, Wu YC, Chou TY, Hsu WH. The prognostic value of the number of negative lymph nodes in esophageal cancer patients after transthoracic resection. Ann Thorac Surg. 2013;96(3):995-1001.

18. Chen Y, Zhang L, Tian J, Ren X, Hao Q. Combining the negative lymph nodes count with the ratio of positive and removed lymph nodes can better predict the postoperative survival in cervical cancer patients. Cancer Cell Int. 2013;13(1):6.

19. Ogino S, Nosho K, Irahara N, et al. Negative lymph node count is associated with survival of colorectal cancer patients, independent of tumoral molecular alterations and lymphocytic reaction. Am J Gastroenterol. 2010;105(2):420-33.

20. Karlsson P, Cole BF, Price KN, et al. The role of the number of uninvolved lymph nodes in predicting locoregional recurrence in breast cancer. J Clin Oncol. 2007;25(15):2019-26.

21. Kaidar-Person O, Lai C, Kuten A, et al. "The Infinite Maze" of breast cancer, signaling pathways and radioresistance. Breast. 2013;22(4):411-8.

22. Eiermann W, Vallis KA. Locoregional treatments for triple-negative breast cancer. Ann Oncol. 2012;23(Suppl 6):vi30-4. 